\title{
New Reactions with Thiosulfines/Dithiiranes: Cycloadditions Leading to Dispiro Derivatives of 1,2,4-Trithiolane
}

\author{
Mohamed I. Hegab, ${ }^{\text {a }}$ Farouk M. E. Abdel-Megeid, ${ }^{\text {b }}$ Farouk A. Gad, ${ }^{\text {b }}$ Sayed A. Shiba, ${ }^{c}$ \\ Inger Søtofte, Jørgen Møller ${ }^{\mathrm{d}}$ and Alexander Senning*,a
}

${ }^{a}$ Department of Applied Chemistry, Technical University of Denmark, DK-2800 Lyngby, Denmark, ${ }^{b}$ National Research Centre, Dokki, Cairo, Egypt, ' ${ }^{C}$ Chemistry Department, Faculty of Science, Ain Shams University, Abassia, Cairo, Egypt, ${ }^{\mathrm{d}}$ Department of Chemistry, Technical University of Denmark, DK-2800 Lyngby, Denmark and department of Chemistry, Odense University, DK-5230 Odense M, Denmark

\begin{abstract}
Hegab, M. I., Abdel-Megeid, F. M. E., Gad, F. A., Shiba, S. A., Søtofte, I., Møller, J. and Senning, A., 1999. New Reactions with Thiosulfines/Dithiiranes: Cycloadditions Leading to Dispiro Derivatives of 1,2,4-Trithiolane. - Acta Chem. Scand. 53: 133-140. (C) Acta Chemica Scandinavica 1999.

The $\beta$-oxo thiosulfines $\mathbf{8}$, generated by 'unzipping' of the corresponding acetyl $\alpha$-chloroalkyl disulfides $\mathbf{1 1}$ with morpholine, are partially converted into the corresponding thioketones $\mathbf{1 2}$ which then cycloadd to $\mathbf{8}$ to give the observed cisand trans-1,2,4-trithiolanes 15. The unsymmetrical Diels-Alder dimerization of 12 plays only a minor role. The new heterocycles thus obtained have been characterized spectroscopically and by X-ray crystallography.
\end{abstract}

Thiosulfines 1 and dithiiranes 2 , cf. eqn. (1), are compounds attracting much topical interest. ${ }^{1-3}$ The generation of thiosulfines/dithiiranes $\mathbf{1} / \mathbf{2}$ from $\alpha$-chloroalkanesulfenyl chlorides $\mathbf{3}$ via acetyl $\alpha$-chloroalkyl disulfides 4 by 'unzipping', eqn. (2), is a convenient and reliable preparative method., ${ }^{4,5}$<smiles>[R]/C(CC)=[SH]/S</smiles><smiles>[Y]C1([R])CS1</smiles>
2

(1)<smiles>[R]C([R])(Cl)SSC(C)=O</smiles><smiles>CC(=O)N1CCOCC1</smiles>

$\beta, \beta^{\prime}$-Dioxo substituted compounds in the form of diaroylmethane derivatives exhibited characteristic behavior in that the otherwise predominant sulfur loss from the corresponding thiosulfine $\mathbf{4}$ was, by and large, suppressed. Furthermore, contrary to all other known $\mathbf{1} / \mathbf{2}$ systems nucleophilic addition of morpholine to give

\footnotetext{
* To whom correspondence should be addressed.
}

5, eqn. (3), was observed which could be explained either in terms of inductive and mesomeric substituent effects on the electron distribution within the thiosulfine moiety (i.e., leading to a preponderance of the resonance contributor 1f, cf. Scheme 1) or by invoking the intermediacy of a tautomer $\mathbf{6}$ formed by intramolecular ring closure of 4 , cf. eqn. (4).

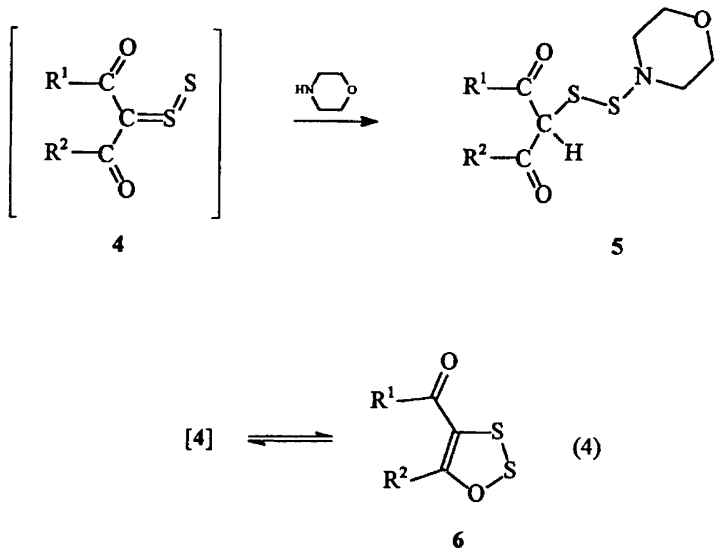

In our present study we wished to examine the generation and reactive behavior of $\beta$-monooxo substituted $\mathbf{1} / \mathbf{2}$ derived from 1-tetralone and its chalcogena analogs, i.e. 8.

The required precursors of 8 , i.e. the ketones 7 , the $\alpha$-chlorosulfenyl chlorides $\mathbf{1 0}$, and the acetyl $\alpha$-chloro- 
alkyl disulfides 11 were prepared according to standard procedures, cf. eqn. (5).<smiles>[R]c1cc([R])c2c(c1[R])C(=O)CC([R])([R])[X]c1c([R])cc([R])c(c1[R])C2=O</smiles>

a, $\mathrm{X}=\mathrm{CH}_{2} ; \mathbf{R}^{\prime}=\mathrm{E} t, \mathrm{R}^{2}=\mathrm{Me}, \mathrm{R}^{3}=\mathrm{R}^{4}=\mathrm{R}^{5}=\mathrm{H}$

b, $X=\mathrm{CH}_{2} ; \mathrm{R}^{\prime}=\mathrm{R}^{2}=\mathrm{R}^{3}=\mathrm{R}^{\mathrm{s}}=\mathrm{Me}, \mathrm{R}^{4}=\mathrm{H}$

c, $\mathrm{X}=\mathrm{CH}_{2} ; \mathrm{R}^{\prime}=\mathrm{R}^{2}=\mathrm{Et}, \mathrm{R}^{3}=\mathrm{R}^{4}=\mathrm{R}^{\mathrm{s}}=\mathrm{H}$

d, $\mathrm{X}=\mathrm{O} ; \mathrm{R}^{1}+\mathrm{R}^{2}=\left(\mathrm{CH}_{2}\right)_{5}, \mathrm{R}^{3}=\mathrm{R}^{4}=\mathrm{R}^{s}=\mathrm{H}$

e, $X=S, R^{\prime}=R^{2}=R^{4}=M c, R^{3}=R^{s}=H$

When 8 was generated according to eqn. (6) it was immediately obvious that the corresponding thioketones 12 were formed as well, presumably via disproportionation, eqn. (7). ${ }^{1.3}$ However, no products derived from the hypothetical thione $S$-disulfides 13 could be identified.

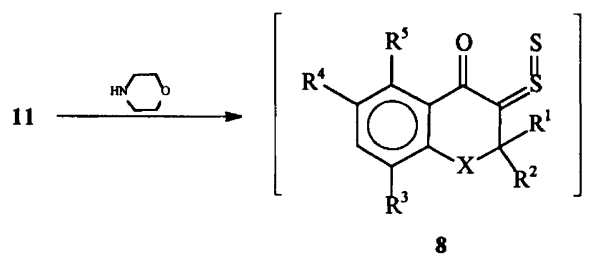

The yields of the cycloadditions following the liberation of 8 according to eqn. (6) are shown in Table 1.

The Diels-Alder dimer of 12, i.e. the spiro compound 14, could be observed in only one case, namely in the shape of 14a, cf. eqn. (8). Such dimers are otherwise known to be formed from $\alpha$-oxo thioketones. ${ }^{8}$

The common denominator of the five reactions examined by us is the formation of the trans-1,2,4-trithiolanes, trans-15, formed from 8 and 12 according to eqn. (9),

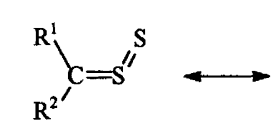

$\mathbf{1 a}$

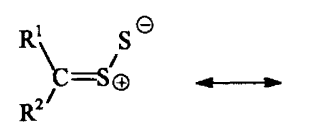

1b<smiles>[R]C([R])[Se]=S</smiles>

1c<smiles>[R]c1cc([R])c([R])c(C(=O)C(=S=S)C([R])([R])[X])c1</smiles><smiles>[R]c1cc([R])c2c(c1[X])[X]C([R])([R])C(=S)C2=O</smiles>

12<smiles>[R]c1cc([R])c2c(c1[X])[X]C([R])([R])C(=S=S)C2=O</smiles>

13
Table 1. Yields (\%) of cycloaddition products from 8.

\begin{tabular}{llll}
\hline Starting compound & $\mathbf{1 4}$ & cis-15 & trans-15 \\
\hline 9a & 0.7 & 2 & 15 \\
$9 \mathrm{~b}$ & - & - & 26 \\
$9 \mathrm{c}$ & - & 2.6 & 20 \\
$9 \mathrm{~d}$ & - & - & 30 \\
$9 \mathrm{e}$ & - & - & 14 \\
\hline
\end{tabular}

cf. Table 1. In two cases, the isomeric cis-15 could be isolated as a minor companion of trans-15. The nature of $\mathrm{X}$ in 8 , i.e. $\mathrm{CH}_{2}(\mathbf{8 a}-\mathrm{c}), \mathrm{O}(\mathbf{8 d})$, or $\mathrm{S}(\mathbf{8 e})$ does not appear to influence the general course of the reactions.

In order to ascertain their cis-trans identity the new sulfur heterocycles obtained in our study were also examined by X-ray crystallography, cf. Figs. 1 and 2. A short summary of the crystal data is given in Table 2 .

The NMR spectra of 15 only partially reflect the stereogenicity of the two carbon atoms of the 1,2,4-trithiolane ring. In the ${ }^{1} \mathrm{H}$ NMR spectra the $\mathrm{CH}_{2}$ protons of the ethyl groups (but not the $\mathrm{CH}_{3}$ protons) exhibit the expected diastereotopy while the ${ }^{13} \mathrm{C}$ NMR spectra fail to show corresponding effects on the carbon atoms.

The EI mass spectra of $\mathbf{1 5}$ all exhibit significant and relatively abundant molecular ion peaks; in the case of

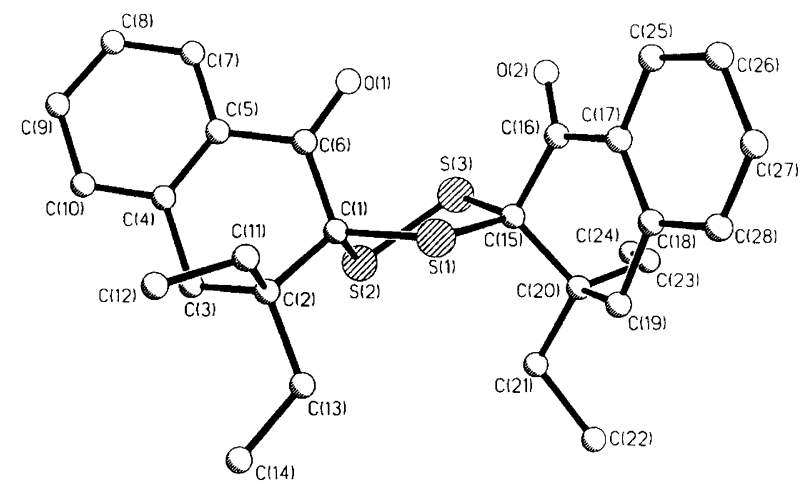

Fig. 1. The structure of cis-15c as determined by $X$-ray crystallography.

Scheme 1. 


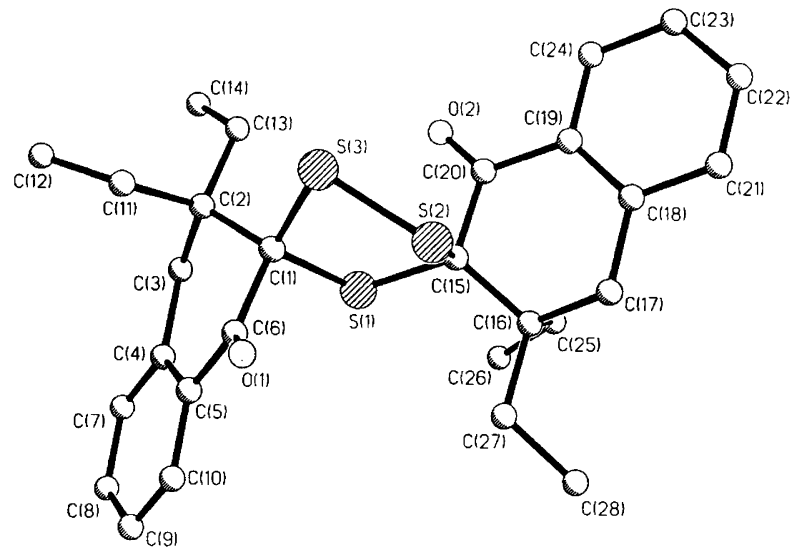

Fig. 2. The structure of trans-15c as determined by X-ray crystallography.

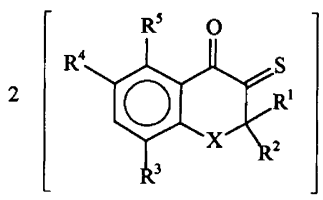

12

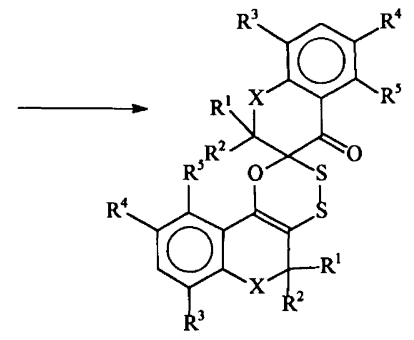

14 trans-15d the $M^{++}$ion gives rise to the base peak. In the EI mass spectra of 15a-c the base peaks correspond to loss of two sulfur atoms from the molecular ion. The EI mass spectra of corresponding cis and trans isomers are very similar and the small differences in the intensities of various ions in, for instance, the 15a isomers do not allow any distinction.

Figure 3 shows the FAB spectra of cis- and trans-15c generated with a 3-nitrobenzyl alcohol matrix. A significant protonated molecular ion $(M+\mathrm{H})^{+}$is present in both spectra at $m / z 497$ as well as peaks at $\mathrm{m} / z 519$ corresponding to the sodiated molecular ions due to the presence of trace amounts of sodium salts in the samples. However, the intensity of this ion relative to that of the protonated molecular ion is much higher in the case of the $c i s$ than of the trans isomer. In addition, the spectrum of $c i s-\mathbf{1 5 c}$ exhibits a peak at $m / z 1015$ corresponding to an $\left(M_{2}+\mathrm{Na}\right)^{+}$ion. No corresponding peak of any significance is seen in the spectrum of trans-15c.

These differences could a priori either be due to unequal amounts of sodium salts in the two samples or to different abilities of these isomers to form such ions. In order to elucidate this point new spectra were recorded with a sodium chloride saturated matrix. The results are shown in Fig. 4. Quite clearly cis-15c is much better able than trans-15c to form sodiated molecular ions as well as $\left(M_{2}+\mathrm{Na}\right)^{+}$ions. Analogous results were obtained with the cis-trans isomers of $\mathbf{1 5 a}$.

Simple geometrical considerations readily lead to the conclusion that the spacing of the two carbonyl oxygen atoms in cis-15 must be conducive to a gas phase $\left(M_{2}+\mathrm{Na}\right)^{+}$ion with a tetracoordinate sodium atom while the geometry of trans-15 only allows the sodium

Table 2. Crystal data for 1,2,4-trithiolanes 15.

\begin{tabular}{|c|c|c|c|c|c|c|c|}
\hline \multirow{2}{*}{$\begin{array}{l}\text { Crystal } \\
\text { data }^{a}\end{array}$} & \multicolumn{7}{|l|}{ Compound } \\
\hline & cis-15a & trans-15a & trans-15b & $c i s-15 c$ & trans-15c & trans-15d & trans-15e \\
\hline $\begin{array}{l}\text { Formula } \\
M_{\mathrm{W}}\end{array}$ & $\begin{array}{l}\mathrm{C}_{26} \mathrm{H}_{28} \mathrm{O}_{2} \mathrm{~S}_{3} \\
468.67\end{array}$ & $\begin{array}{l}\mathrm{C}_{26} \mathrm{H}_{28} \mathrm{O}_{2} \mathrm{~S}_{3} \\
468.67\end{array}$ & $\begin{array}{l}\mathrm{C}_{28} \mathrm{H}_{32} \mathrm{O}_{2} \mathrm{~S}_{3} \\
496.73\end{array}$ & $\begin{array}{l}\mathrm{C}_{28} \mathrm{H}_{32} \mathrm{O}_{2} \mathrm{~S}_{3} \\
496.73\end{array}$ & $\begin{array}{l}\mathrm{C}_{28} \mathrm{H}_{32} \mathrm{O}_{2} \mathrm{~S}_{3} \\
496.73\end{array}$ & $\begin{array}{l}\mathrm{C}_{28} \mathrm{H}_{28} \mathrm{O}_{4} \mathrm{~S}_{3} \\
524.68\end{array}$ & $\begin{array}{l}\mathrm{C}_{24} \mathrm{H}_{24} \mathrm{O}_{2} \mathrm{~S}_{5} \\
504.73\end{array}$ \\
\hline M.p. & $167-170^{\circ} \mathrm{C}$ & $162-165^{\circ} \mathrm{C}$ & $218-220^{\circ} \mathrm{C}$ & $148-150^{\circ} \mathrm{C}$ & $190-192^{\circ} \mathrm{C}$ & $280-282^{\circ} \mathrm{C}$ & $198-201^{\circ} \mathrm{C}$ \\
\hline Crystal system & Monoclinic & Monoclinic & Monoclinic & Monoclinic & $\begin{array}{l}\text { Triclinic } \\
P \overline{1}\end{array}$ & Tetragonal & Monoclinic \\
\hline $\begin{array}{l}\text { Space group } \\
a / \AA\end{array}$ & $\begin{array}{l}P 2_{1} / c \\
11.0967(5)\end{array}$ & $\begin{array}{l}P 2_{1} / c \\
10.1910(4)\end{array}$ & $\begin{array}{l}P 2_{1} / c \\
18.8658(2)\end{array}$ & $\begin{array}{l}P 2_{1} / n \\
8.8167(2)\end{array}$ & $\begin{array}{l}P 1 \\
9.8988(3)\end{array}$ & $\begin{array}{l}P 4_{3} 2,2 \\
10.7929(2)\end{array}$ & $\begin{array}{l}P 2_{1} / n \\
11.7934(2)\end{array}$ \\
\hline$b / \AA$ & $16.8755(8)$ & $36.5703(14)$ & $11.1475(2)$ & $10.88230(10)$ & $10.1076(3)$ & $10.7929(2)$ & $17.4284(3)$ \\
\hline$c / \AA$ & $12.4165(6)$ & $12.7772(5)$ & $12.1351(2)$ & $26.5226(6)$ & $14.4903(4)$ & $21.8689(4)$ & $12.1621(1)$ \\
\hline$\alpha /^{\circ}$ & - & - & - & - & $78.2660(1)$ & - & - \\
\hline$\beta /{ }^{\circ}$ & $99.0780(10)$ & $90.430(2)$ & $92.0880(10)$ & $90.7410(10)$ & $80.5360(1)$ & - & $107.812(1)$ \\
\hline$\gamma /^{\circ}$ & - & - & - & - & $62.967(1)$ & - & - \\
\hline$V / A^{3}$ & $2296.0(2)$ & $4761.8(3)$ & $2550.40(7)$ & $2544.52(8)$ & $1260.10(6)$ & $2547.44(8)$ & 2379.97(6) \\
\hline$Z$ & 4 & 8 & 4 & 4 & 2 & 4 & 4 \\
\hline \multicolumn{8}{|l|}{ Total number } \\
\hline of unique refl. & 4906 & 8349 & 6454 & 6446 & 6058 & 3389 & 6033 \\
\hline$[I>2 \sigma(I)]$ & 3708 & 4197 & 5370 & 4130 & 5436 & 2616 & 4732 \\
\hline$\theta$-range $/^{\circ}$ & $1.86-27.00$ & $1.11-25.00$ & $1.08-29.71$ & $1.54-29.57$ & $1.44-29.62$ & $2.10-29.66$ & $2.11-29.58$ \\
\hline$R$ (obs. data) & 0.0810 & 0.1192 & 0.0386 & 0.0702 & 0.0381 & 0.0500 & 0.0406 \\
\hline$w R 2$ (all data) & 0.1863 & 0.2545 & 0.1140 & 0.1544 & 0.1013 & 0.0958 & 0.1080 \\
\hline
\end{tabular}

${ }^{a}$ The data were collected at $296 \mathrm{~K}$ on a SMART diffractometer using Mo K $\alpha$ radiation. The crystal-to-detector distance was $4.5 \mathrm{~cm}$. The structures were solved by direct methods (SHELXTL) and refined with a full-matrix least-squares technique. 

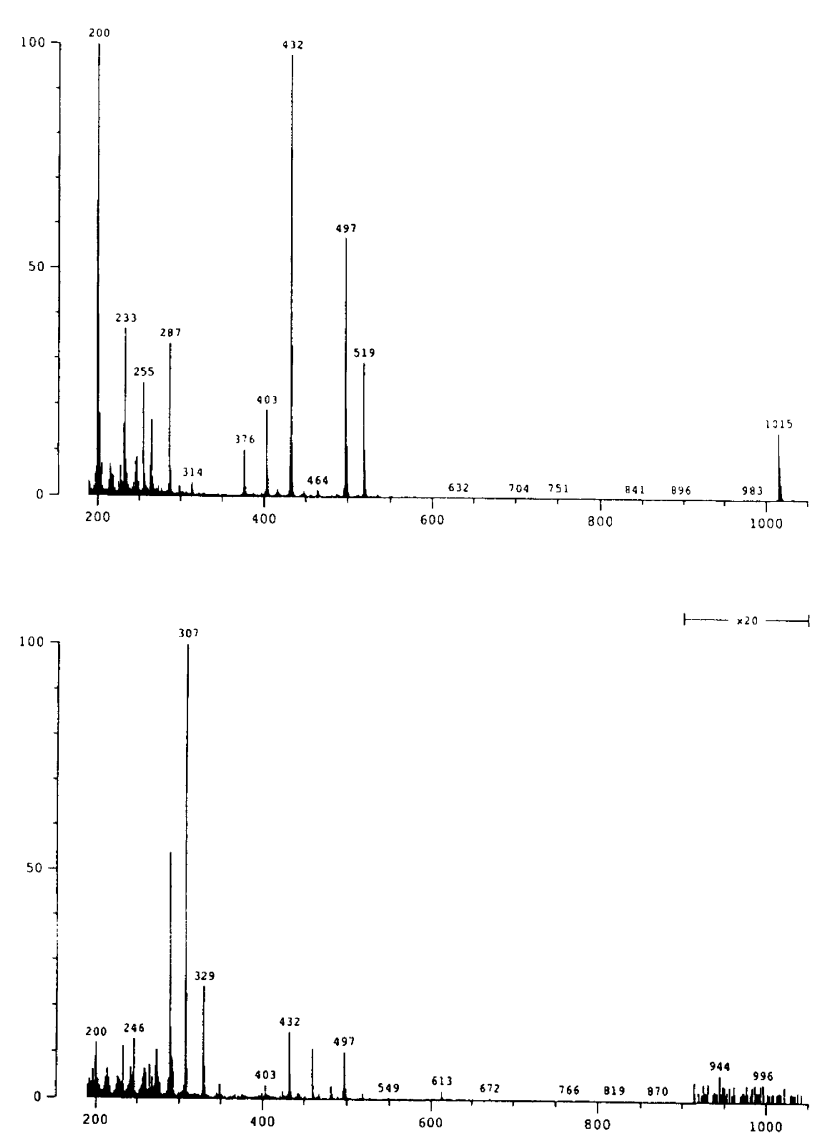

Fig. 3. The FAB mass spectra of cis-15c (top) and of trans$15 c$ (bottom).

atom to become dicoordinate. Analogous differences are to be expected for the sodiated molecular ions.

\section{Experimental}

NMR spectra were taken for samples in $\mathrm{CDCl}_{3}$ with TMS as an internal standard with a Bruker AC 250 or Bruker AM 500 apparatus. EI $(70 \mathrm{eV})$ mass spectra were recorded on a Finnigan SSQ 710 quadrupole mass spectrometer by direct inlet. FAB mass spectra were obtained with a Kratos MS50 RF instrument with 3-nitrobenzyl alcohol as the matrix and $9 \mathrm{keV} \mathrm{Xe}$ atoms. IR spectra were obtained with a Perkin Elmer 1600 Series FTIR instrument for neat samples (for liquids) or $\mathrm{KBr}$ wafers (for solids).

Elemental analyses were performed by the Microanalytical Laboratory of the Department of Physical Chemistry, University of Vienna, A-1090 Vienna, Austria and by Institute of Organic Chemistry, Siberian Division of the Russian Academy of Sciences, RUS-664033 Irkutsk, Russia. The known compounds 9b, ${ }^{8} 9 d,{ }^{9,13} 9 e,{ }^{10} 10 b,{ }^{11} 10 d,{ }^{12}$ and $10 e^{12}$ were prepared according to literature procedures. The single crystals for the X-ray work were obtained by slow evaporation of the corresponding hexane-ether $(5: 1)$ elutes from the column chromatographic separations.
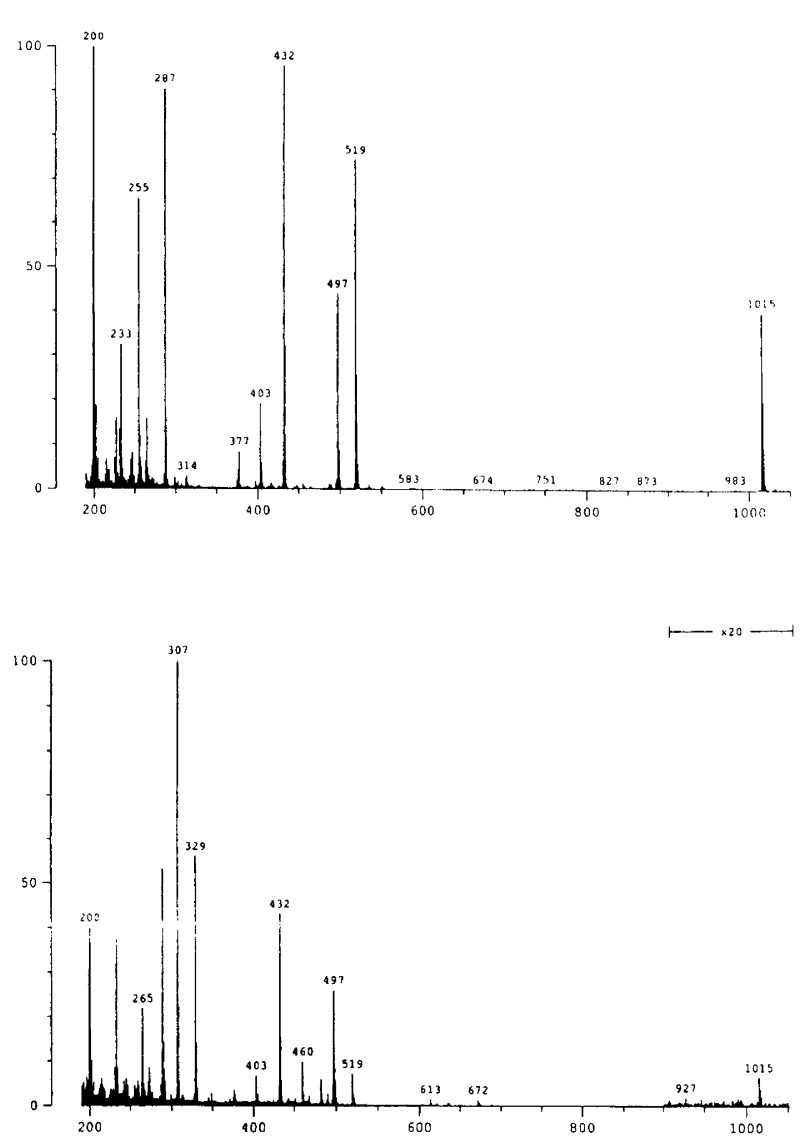

Fig. 4. The $\mathrm{NaCl}$ saturated $\mathrm{FAB}$ mass spectra of cis-15c (top) and of trans-15c (bottom).

3,3-Diethyl-1,2,3,4-tetrahydronaphthalen-1-one 9c. A general procedure for the synthesis of substituted tetralones ${ }^{6}$ was followed. Ethyl $\alpha$-cyano- $\beta, \beta$-diethylacrylate ${ }^{14}$ $(158.0 \mathrm{~g}, 0.77 \mathrm{~mol})$, dissolved in $150 \mathrm{ml}$ dry ether, was added over $45 \mathrm{~min}$ at room temperature to a Grignard solution prepared from $103.7(0.90 \mathrm{~mol})$ benzyl chloride and $22.0 \mathrm{~g}(0.90 \mathrm{~mol})$ magnesium turnings in $115 \mathrm{ml}$ dry ether. When the spontaneous reflux had subsided the mixture was stirred and heated to reflux for another $1 \mathrm{~h}$. The reaction mixture was cooled and poured onto $500 \mathrm{~g}$ cracked ice and then acidified with $20 \%$ sulfuric acid. After the usual work-up the combined ether phases were washed successively with $125 \mathrm{ml}$ water and $125 \mathrm{ml}$ saturated brine, filtered through a layer of anhydrous sodium sulfate, evaporated, and the residue distilled in vacuo. Yield of ethyl 3-benzyl-2-cyano-3-ethylpentanoate $165 \mathrm{~g}$ (79\%), b.p. $155-165^{\circ} \mathrm{C} / 1.5 \mathrm{mmHg}$ (bath temperature $200-210^{\circ} \mathrm{C}$ ). IR (neat): $v_{\mathrm{C}=\mathrm{N}}=2246, v_{\mathrm{C}=\mathrm{O}}=1738 \mathrm{~cm}^{-1}$. ${ }^{1} \mathrm{H} \quad$ NMR $(500 \mathrm{MHz}): \delta=0.92-0.99(6 \mathrm{H}, \mathrm{m}, 3-$ $\left.\mathrm{CH}_{3} \mathrm{CH}_{2}+5-\mathrm{CH}_{3}\right), 1.27\left(3 \mathrm{H}, \mathrm{t}, \mathrm{CH}_{3} \mathrm{CH}_{2} \mathrm{O}\right), 1.49-1.71$ $\left(4 \mathrm{H}, \mathrm{m}, 3-\mathrm{CH}_{3} \mathrm{CH}_{2}+4-\mathrm{CH}_{2}\right), 2.79(1 \mathrm{H}, \mathrm{d}$, $\left.3-\mathrm{C}_{6} \mathrm{H}_{5} \mathrm{CH}_{\mathrm{a}} \mathrm{H}_{\mathrm{b}}\right), 2.85\left(1 \mathrm{H}, \mathrm{d}, 3-\mathrm{C}_{6} \mathrm{H}_{5} \mathrm{CH}_{\mathrm{a}} \mathrm{H}_{\mathrm{b}}\right), 3.41$ $(1 \mathrm{H}, \mathrm{s}, 2-\mathrm{CH}), 4.19\left(2 \mathrm{H}, \mathrm{q}, \mathrm{CH}_{3} \mathrm{CH}_{2} \mathrm{O}\right), 7.19-7.28$ ( $5 \mathrm{H}, \mathrm{m}, 5 \mathrm{ArH})$. MS (EI): $m / z(\%) 273(M, 2), 228(4)$, 200 (3), 160 (100), 131 (17), 91 (62). Anal. Calcd. for 
$\mathrm{C}_{17} \mathrm{H}_{23} \mathrm{NO}_{2}$ (273.25): C, 74.65, H, 8.48, N, 5.12. Found: C, 74.48, H, 8.26, N, 5.06.

Ethyl 3-benzyl-2-cyano-3-ethylpentanoate (172 g, $0.63 \mathrm{~mol})$ was mixed with a solution of $67.0 \mathrm{~g}(1.0 \mathrm{~mol})$ potassium hydroxide in $360 \mathrm{ml}$ ethylene glycol and heated under reflux for $3 \mathrm{~h}$ (the reflux condenser was equipped with a rubber stopper). The cooled reaction mixture was diluted with $400 \mathrm{ml}$ water and then extracted successively with 250,100 , and $100 \mathrm{ml}$ ether. The combined ether extracts were washed with $100 \mathrm{ml}$ water and $100 \mathrm{ml}$ saturated brine and then filtered through a layer of anhydrous sodium sulfate. The solvent was evaporated off and the residue distilled in vacuo. This yielded $109 \mathrm{~g}$ (86\%) 3-benzyl-3-ethylpentanenitrile, b.p. $125-132^{\circ} \mathrm{C} /$ $2 \mathrm{mmHg}$. IR (neat): $v_{\mathrm{C}=\mathrm{N}}=2243 \mathrm{~cm}^{-1}$. MS (EI): $\mathrm{m} / z$ (\%) $201(M, 38), 110(9), 92(80), 91$ (100). Anal. Calc. for $\mathrm{C}_{14} \mathrm{H}_{19} \mathrm{~N}$ (201.29): C, 83.53, H, 9.51, N, 6.95. Found: $\mathrm{C}, 83.66, \mathrm{H}, 9.43, \mathrm{~N} 6.81$. This nitrile was converted, by being heated to reflux with a 3-molar excess of $\mathrm{KOH}$ and ethylene glycol for $6 \mathrm{~h}$ and subsequent conventional work-up, to give 3-benzyl-3-ethylpentanoic acid, b.p. $165-170^{\circ} \mathrm{C} / 2 \mathrm{mmHg}$, yield $88 \%$. IR (neat): $v_{\mathrm{OH}}=$ $3250-3650, v_{\mathrm{C}=\mathrm{O}}=1718 \mathrm{~cm}^{-1} .{ }^{1} \mathrm{H}$ NMR $(250 \mathrm{MHz}): \delta=$ $0.91\left(6 \mathrm{H}, \mathrm{t}, 3-\mathrm{CH}_{3} \mathrm{CH}_{2}+5-\mathrm{CH}_{3}\right), 1.31-1.55(4 \mathrm{H}, \mathrm{m}$, $\left.3-\mathrm{CH}_{3} \mathrm{CH}_{2}+4-\mathrm{CH}_{2}\right), 2.25\left(2 \mathrm{H}, \mathrm{s}, 2-\mathrm{CH}_{2}\right), 2.65(2 \mathrm{H}, \mathrm{s}$, $\left.3-\mathrm{C}_{6} \mathrm{H}_{5} \mathrm{CH}_{2}\right), 7.11-7.29$ ( $\left.5 \mathrm{H}, \mathrm{m}, 5 \mathrm{ArH}\right), \mathrm{MS}(\mathrm{EI}): \mathrm{m} / z$ (\%) $220(M, 61), 131(27), 128$ (68), 92 (76), 91 (100), 83 (35), 69 (53). Anal. Calc. for $\mathrm{C}_{14} \mathrm{H}_{20} \mathrm{O}_{2}$ (220.30): C, 76.32, H, 9.15. Found: C, 75.87, H, 8.95.

Polyphosphoric acid (PPA) (250 g) was heated, on a steam bath, to $90{ }^{\circ} \mathrm{C}$. Then it was removed from the steam bath and $99.0 \mathrm{~g}(0.45 \mathrm{~mol})$ 3-benzyl-3-ethylpentanoic acid, preheated to $65^{\circ} \mathrm{C}$, added at once, and the reaction mixture stirred for $3 \mathrm{~min}$. It was then placed on a steam bath, another $150 \mathrm{~g}$ PPA were added, stirring was continued for $30 \mathrm{~min}$, and the temperature was maintained at $90^{\circ} \mathrm{C}$. After being cooled to room temperature the reaction mixture was poured, with stirring, into ice-water. A viscous brown oil precipitated and soon changed color to yellowish green. Subsequently, the crude product was extracted with ether (three times) and the combined ether extracts successively washed with $300 \mathrm{ml}$ water, $2 \times 200 \mathrm{ml} 5 \%$ aqueous $\mathrm{NaOH}, 300 \mathrm{ml}$ water, $200 \mathrm{ml} \mathrm{3 \%}$ aqueous acetic acid, and $100 \mathrm{ml}$ water. The organic phase was dried over magnesium sulfate, filtered, and evaporated. The residue was vacuum distilled to yield $60.0 \mathrm{~g}(71 \%)$, b.p. $145-155^{\circ} \mathrm{C} / 3 \mathrm{mmHg}$, yield $71 \%$. IR (neat): $v_{\mathrm{C}=0}=1677 \mathrm{~cm}^{-1} .{ }^{1} \mathrm{H}$ NMR $(250 \mathrm{MHz}): \delta=$ $0.85\left[6 \mathrm{H}, \mathrm{t}, 3,3-\left(\mathrm{CH}_{3} \mathrm{CH}_{2}\right)_{2}\right], 1.42[4 \mathrm{H}, \quad \mathrm{q}, 3,3-$ $\left.\left(\mathrm{CH}_{3} \mathrm{CH}_{2}\right)_{2}\right], 2.49\left(2 \mathrm{H}, \mathrm{s}, 2-\mathrm{CH}_{2}\right), 2.84\left(2 \mathrm{H}, \mathrm{s}, 4-\mathrm{CH}_{2}\right)$, 7.25-7.98 (4 H, m, 4 Ar-H). ${ }^{13} \mathrm{C}$ NMR (125.7 MHz): $\delta=7.59\left(2 \times 3-\mathrm{CH}_{3} \mathrm{CH}_{2}\right), 28.66\left(2 \times 3-\mathrm{CH}_{3} \mathrm{CH}_{2}\right), 38.88$ (C-3), 39.20 (C-4), 48.77 (C-2), 126.38, 126.47, 129.30, 132.22, 133.60, 142.38 (C-4a, C-5, C-6, C-7, C-8, C-8a), 198.75 (C-1). MS (EI): $m / z$ (\%) $202(M, 67), 173$ $\left(M-\mathrm{C}_{2} \mathrm{H}_{5}, 70\right), 145(30), 131(95), 118\left(\mathrm{C}_{8} \mathrm{H}_{6} \mathrm{O}, 100\right)$, $90\left(\mathrm{C}_{7} \mathrm{H}_{6}, 22\right)$. Found: $\mathrm{C}, 82.91 ; \mathrm{H}, 9.16$; calc. for $\mathrm{C}_{14} \mathrm{H}_{18} \mathrm{O}$ (202.28): C, 83.12; $\mathrm{H}, 8.96 \%$.
(RS) -3-Ethyl-1,2,3,4-tetrahydro-3-methylnaphthalen-1one 9a, b.p. 134-136/4 mmHg), yield $71 \%$. IR (neat): $v_{\mathrm{C}=\mathrm{O}}=1684 \mathrm{~cm}^{-1} .{ }^{1} \mathrm{H}$ NMR $(250 \mathrm{MHz}): \delta=0.95(3 \mathrm{H}$, t, $\left.\mathrm{CH}_{3} \mathrm{CH}_{2}\right), 1.04(3 \mathrm{H}, \mathrm{s}, 3-\mathrm{Me}), 1.43\left(2 \mathrm{H}, \mathrm{q}, \mathrm{CH}_{3} \mathrm{CH}_{2}\right)$, $2.46\left(1 \mathrm{H}, \mathrm{d}, 2-\mathrm{CH}_{\mathrm{a}} \mathrm{H}_{\mathrm{b}}\right), 2.55\left(1 \mathrm{H}, \mathrm{d}, 2-\mathrm{CH}_{\mathrm{a}} H_{\mathrm{b}}\right), 2.75$ $\left(1 \mathrm{H}, \mathrm{d}, 4-\mathrm{CH}_{\mathrm{a}} \mathrm{H}_{\mathrm{b}}\right), 2.91\left(1 \mathrm{H}, \mathrm{d}, 4-\mathrm{CH}_{\mathrm{a}} H_{\mathrm{b}}\right), 7.18-7.25$ (1 H, m, Ar-H), 7.29-7.37 (1 H, m, Ar-H), 7.46-7.55 (1 H, m, Ar-H), 7.98-8.08 (1 H, m, Ar-H). ${ }^{13} \mathrm{C}$ NMR (62.9 MHz): $\delta=7.94\left(\mathrm{CH}_{3} \mathrm{CH}_{2}\right), 24.19\left(3-\mathrm{CH}_{3}\right), 33.31$ $\left(\mathrm{CH}_{3} \mathrm{CH}_{2}\right), 36.33(\mathrm{C}-3), 41.27(\mathrm{C}-4), 50.60(\mathrm{C}-2), 126.38$, 128.21, 129.25, 131.88, 133.55, 142.44 (C-4a, C-5, C-6, C-7, C-8, C8a), 198.64 (C-1). MS (EI): $m / z(\%) 188$ ( $M$, 68), $173\left(M-\mathrm{CH}_{3}, 10\right), 159\left(M-\mathrm{C}_{2} \mathrm{H}_{5}, 44\right), 131$ (42), $118\left(\mathrm{C}_{8} \mathrm{H}_{6} \mathrm{O}, 100\right), 90$ (24). Found: $\mathrm{C}, 82.80 ; \mathrm{H}, 8.29$; calc. for $\mathrm{C}_{13} \mathrm{H}_{16} \mathrm{O}$ (188.26): C, 82.98; $\mathrm{H}, 8.51 \%$.

Sulfenyl chlorides 10. A general procedure for the conversion of 3,3-dialkyl-1-tetralones to $\alpha$-chlorosulfenyl chlor$\operatorname{ides}^{8}$ was followed. The ketone $9(0.10 \mathrm{~mol})$ was dissolved in $37 \mathrm{ml}$ thionyl chloride. The temperature rose to $35^{\circ} \mathrm{C}$ and a brisk evolution of gas started after $2 \mathrm{~min}$. The reaction mixture was allowed to stand for $3 \mathrm{~h}$ at room temperature. Excess thionyl chloride was removed on a rotatory evaporator to leave crude $\mathbf{1 0}$ which was then recrystallized from ligroin (b.p. $80-100^{\circ} \mathrm{C}$ ).

(2RS,3SR) - 2 - Chloro - 3- ethyl - 1,2,3,4 - tetrahydro -3 methyl-1-oxonaphthalene-2-sulfenyl chloride (2RS,3SR)10a. M.p. $115-117^{\circ} \mathrm{C}$, yield $43 \%$ (crude yield $65 \%$ ). IR $(\mathrm{KBr}): v_{\mathrm{C}=\mathrm{O}}=1692 \mathrm{~cm}^{-1} .{ }^{1} \mathrm{H}$ NMR $(250 \mathrm{MHz}): \delta=1.05$ ( $\left.3 \mathrm{H}, \mathrm{t}, \mathrm{CH}_{3} \mathrm{CH}_{2}\right), 1.19$ (3 H, s, 3-Me), 1.69-1.87 (1 H, $\left.\mathrm{m}, \mathrm{CH}_{3} \mathrm{CH}_{\mathrm{a}} \mathrm{H}_{\mathrm{b}}\right), 2.08-2.25\left(1 \mathrm{H}, \mathrm{m}, \mathrm{CH}_{3} \mathrm{CH}_{\mathrm{a}} H_{\mathrm{b}}\right), 3.04$ $\left(1 \mathrm{H}, \mathrm{d}, 4-\mathrm{CH}_{\mathrm{a}} \mathrm{H}_{\mathrm{b}}\right), 3.20\left(1 \mathrm{H}, \mathrm{d}, 4-\mathrm{CH}_{\mathrm{a}} H_{\mathrm{b}}\right), 7.18-7.27$ (1 H, m, ArH), 7.35-7.42 (1 H, m, ArH), 7.51-7.61 (1 H, m, ArH ), 8.11-8.19 (1 H, m, ArH ). ${ }^{13} \mathrm{C}$ NMR (62.9 MHz): $\delta=7.78\left(\mathrm{CH}_{3} \mathrm{CH}_{2}\right), 20.50\left(3-\mathrm{CH}_{3}\right), 30.84$ $\left(\mathrm{CH}_{3} \mathrm{CH}_{2}\right), 39.32(\mathrm{C}-4), 47.25(\mathrm{C}-3), 94.15(\mathrm{C}-2), 127.26$, $128.80,129.17,129.17,134.16,138.83$ (C-4a, C-5, C-6, C-7, C-8, C8a), 184.22 (C-1). MS (EI): $m / z(\%) 288(M$, 33), 253 (M-Cl, 56), 217 (34), 185 (39), 157 (38), 152 (100), 128 (45), $118\left(\mathrm{C}_{8} \mathrm{H}_{6} \mathrm{O}, 62\right), 115$ (39), 90 (56). Found: C, 54.29; H, 4.85; Cl, 24.31; S, 11.15; calc. for $\mathrm{C}_{13} \mathrm{H}_{14} \mathrm{Cl}_{2} \mathrm{OS}(289.21): \mathrm{C}, 53.98 ; \mathrm{H}, 4.88 ; \mathrm{Cl}, 24.51 ; \mathrm{S}$, $11.08 \%$. The corresponding ( $2 R S, 3 R S)-10 \mathrm{a}$ isomer could be observed in the crude product, but even repeated, painstaking column chromatography failed to provide a pure sample.

2-Chloro-3,3-diethyl-1,2,3,4-tetrahydro-1-oxonaphthalene-2-sulfenyl chloride 10c. M.p. $52-55^{\circ} \mathrm{C}$, yield $48 \%$. IR (KBr): $v_{\mathrm{C}=\mathrm{O}}=1698 \mathrm{~cm}^{-1} ;{ }^{1} \mathrm{H}$ NMR $(250 \mathrm{MHz}): \delta=$ $0.82,1.15\left(6 \mathrm{H}, \mathrm{t}, 3,3-\mathrm{CH}_{3} \mathrm{CH}_{2}\right), 1.38-2.19(4 \mathrm{H}, \mathrm{m}, 3,3-$ $\left.\mathrm{CH}_{3} \mathrm{CH}_{2}\right), 3.01-3.27\left(2 \mathrm{H}, \mathrm{q}, 4-\mathrm{CH}_{2}\right), 7.23(1 \mathrm{H}, \mathrm{d}$, ArH ), 7.38 (1 H, t , ArH ), 7.58 (1 H, t , ArH ), $8.15(1 \mathrm{H}$, d, ArH ). ${ }^{13} \mathrm{C}$ NMR (62.9 MHz): $\delta=9.43\left(3-\mathrm{CH}_{3} \mathrm{CH}_{2}\right)$, $9.50 \quad\left(3-\mathrm{CH}_{3} \mathrm{CH}_{2}\right), \quad 27.61 \quad\left(3-\mathrm{CH}_{3} \mathrm{CH}_{2}\right), \quad 30.25$ (3- $\mathrm{CH}_{3} \mathrm{CH}_{2}$ ), 36.79 (C-4), 49.36 (C-3), 94.49 (C-2), $127.19,128.37,129.02,129.61,134.14,138.92$ (C-4a, C-5, 
C-6, C-7, C-8, C8a), 183.83 (C-1). MS (EI): $m / z$ (\%) 302 $(M, 26), 267(M-\mathrm{Cl}, 65), 231(24), 199(59), 171$ (47), 152 (100), $149(62), 128(53), 118\left(\mathrm{C}_{8} \mathrm{H}_{6} \mathrm{O}, 75\right)$. Found: $\mathrm{C}, 55.52 ; \mathrm{H}, 5.28 ; \mathrm{Cl}, 23.44 ; \mathrm{S}, 10.73$; calc. for $\mathrm{C}_{14} \mathrm{H}_{16} \mathrm{Cl}_{2} \mathrm{OS}(303.24): \mathrm{C}, 55.44 ; \mathrm{H}, 5.31 ; \mathrm{Cl}, 23.38$; $\mathrm{S}, 10.57 \%$.

Acetyl $\alpha$-chloroalkyl disulfides 11. A general procedure for the conversion of $\alpha$-chlorosulfenyl chlorides to acetyl $\alpha$-chloroalkyl disulfides ${ }^{4}$ was followed. Thioacetic acid $(0.8 \mathrm{ml}, 0.01 \mathrm{~mol})$ was added to a solution of $0.01 \mathrm{~mol} 9$ in $30 \mathrm{ml} \mathrm{CCl}_{4}$ and the reaction mixture kept at $50-60{ }^{\circ} \mathrm{C}$ until completion of the reaction as judged by TLC $(3 \mathrm{~h})$. The solvent was then evaporated off and the oily residue treated with ligroin (b.p. $90-100^{\circ} \mathrm{C}$ ) until solidification. This crude solid was then recrystallized from ligroin (b.p. $90-100^{\circ} \mathrm{C}$ ).

Acetyl (2RS,3SR)-2-chloro-3-ethyl-1,2,3,4-tetrahydro-3methyl-1-oxonaphthalen-2-yl disulfide 11a. M.p. $86-88^{\circ} \mathrm{C}$ (from petroleum ether, b.p. $40-60{ }^{\circ} \mathrm{C}$ ), yield $60 \%$. IR $(\mathrm{KBr}): v_{\mathrm{C}=\mathrm{O}}=1692 \mathrm{~cm}^{-1} .{ }^{1} \mathrm{H}$ NMR $(250 \mathrm{MHz}): \delta=0.89$ (3 H. s. 3-Me $) .1 .13$ (3 H. s. 3-Me $\mathrm{Me}_{\mathrm{b}}$ ) $1.35-1.53(1 \mathrm{H}$, $\left.\mathrm{m}, \mathrm{CH}_{3} \mathrm{CH}_{\mathrm{a}} \mathrm{H}_{\mathrm{b}}\right), 1.79-2.21\left(1 \mathrm{H}, \mathrm{m}, \mathrm{CH}_{3} \mathrm{CH}_{\mathrm{a}} H_{\mathrm{b}}\right), 2.48$ $\left(3 \mathrm{H}, \mathrm{s}, \mathrm{CH}_{3} \mathrm{CO}\right), 3.09\left(1 \mathrm{H}, \mathrm{d}, \mathrm{PhCH}_{\mathrm{a}} \mathrm{H}_{\mathrm{b}}\right), 3.47(1 \mathrm{H}$, d, $\left.\mathrm{PhCH}_{\mathrm{a}} H_{\mathrm{b}}\right), 7.21-7.31(1 \mathrm{H}, \mathrm{m}, \mathrm{ArH}), 7.41(1 \mathrm{H}, \mathrm{t}$, $\mathrm{ArH}), 7.61$ (1 H, t, ArH ), $7.61(1 \mathrm{H}, \mathrm{d}, \mathrm{ArH}) .{ }^{13} \mathrm{C}$ NMR $(125.7 \mathrm{MHz}): \delta=7.80 \quad\left(3-\mathrm{CH}_{3} \mathrm{CH}_{2}\right), 20.60 \quad\left(3-\mathrm{CH}_{3}\right)$, $27.75\left(\mathrm{CH}_{3} \mathrm{CO}\right), 28.89\left(3-\mathrm{CH}_{3} \mathrm{CH}_{2}\right), 37.33(\mathrm{C}-4), 46.58$ (C-3), 92.64 (C-2), 127.12, 127.18, 128.42, 128.96, 134.00, 138.69, (C-4a, C-5, C-6, C-7, C-8, C-8a), 184.12 (C-1), $191.50\left(\mathrm{CH}_{3} \mathrm{CO}\right)$. MS (EI): $m / z(\%) 264\left(M-\mathrm{S}_{2}, 29\right)$, $222\left(m / z 264-\mathrm{CH}_{2} \mathrm{CO}, 33\right), 193\left(m / z 222-\mathrm{C}_{2} \mathrm{H}_{5}, 100\right)$, 157 (14), 43 (20). Found: C, 55.09; H, 5.24; Cl, 10.79; S, 19.30; calc. for $\mathrm{C}_{15} \mathrm{H}_{17} \mathrm{ClO}_{2} \mathrm{~S}_{2}$ (328.86): C, 54.78; $\mathrm{H}$, $5.21 ; \mathrm{Cl}, 10.78 ; \mathrm{S}, 19.46 \%$.

Acetyl (RS)-2-chloro-1,2,3,4-tetrahydro-3,3,5,8-tetramethyl-1-oxonaphthalen-2-yl disulfide 11b. M.p. $118-120^{\circ} \mathrm{C}$ (from ether-hexane), yield $91 \%$. IR ( $\mathrm{KBr}$ ): $v_{\mathrm{C}=\mathrm{O}}=1693 \mathrm{~cm}^{-1} .{ }^{1} \mathrm{H}$ NMR $(500 \mathrm{MHz}): \delta=1.23(3 \mathrm{H}$, s, 3-Me), 1.59 (3 H, s, 3-Me), 2.28 (3 H, s, 5-Me), 2.37 ( $3 \mathrm{H}, \mathrm{s}, 8-\mathrm{Me}), 2.56(3 \mathrm{H}, \mathrm{s}, \mathrm{MeCO}), 2.88\left(1 \mathrm{H}, \mathrm{d}, 4-\mathrm{H}_{\mathrm{a}}\right)$, $3.22\left(1 \mathrm{H}, \mathrm{d}, 4-\mathrm{H}_{\mathrm{b}}\right), 7.13(1 \mathrm{H}, \mathrm{d}, \operatorname{ArH}), 7.29(1 \mathrm{H}, \mathrm{d}$, $\mathrm{ArH}) .{ }^{13} \mathrm{C}$ NMR $(125.7 \mathrm{MHz}): \delta=19.39\left(5-\mathrm{CH}_{3}\right), 22.99$ $\left(8-\mathrm{CH}_{3}\right), 25.91,25.12\left(2 \times 3-\mathrm{CH}_{3}\right), 28.38\left(\mathrm{CH}_{3} \mathrm{CO}\right), 40.72$ (C-4), 42.79 (C-3), 93.41 (C-2), 127.83, 130.39, 133.77, 134.44, 138.05, 140.52 (C-4a, C-5, C-6, C-7, C-8, C-8a), 186.58 (C-1), $192.33\left(\mathrm{CH}_{3} \mathrm{CO}\right)$. MS (EI): $m / z(\%) 342$ $(M, 0.3), 278\left(M-\mathrm{S}_{2}, 55\right), 236\left(m / z 278-\mathrm{CH}_{2} \mathrm{CO}, 82\right)$, $221\left(\mathrm{~m} / \mathrm{z} 236-\mathrm{CH}_{3}, 100\right), 185$ (4), 43 (25). Found: C, $56.35 ; \mathrm{H}, 5.68 ; \mathrm{Cl}, 10.49 ; \mathrm{S}, 18.59$; calc. for $\mathrm{C}_{16} \mathrm{H}_{19} \mathrm{ClO}_{2} \mathrm{~S}_{2}$ (342.88): C, 56.04; H, 5.58; Cl, 10.34; S, 18.70\%.

Acetyl 2-chloro-3,3-diethyl-1,2,3,4-tetrahydro-1-oxonaphthalen-2-yl disulfide 11c. The crude product obtained from 10c and thioacetic acid was used immediately for the reaction with morpholine (vide infra).
Acetyl 3'-chloro-4'-oxospiro[cyclohexane-1,2'-chroman]$3^{\prime}-y$ l disulfide 11d. M.p. $132-133^{\circ} \mathrm{C}$ (from ether), yield $89 \%$. IR ( KBr): $v_{\mathrm{C}=\mathrm{O}}=1692 \mathrm{~cm}^{-1} .{ }^{1} \mathrm{H}$ NMR $(250 \mathrm{MHz})$ : $\delta=1.18-2.62\left(10 \mathrm{H}, \mathrm{m}, 5 \mathrm{CH}_{2}\right), 2.51\left(3 \mathrm{H}, \mathrm{s}, \mathrm{CH}_{3} \mathrm{CO}\right)$, 6.99-7.17 (2 H, m, 2 ArH), 7.52-7.62 (1 H, m, ArH), 7.85-7.95 (1 H, dd, ArH ). ${ }^{13} \mathrm{C}$ NMR (62.9 MHz): $\delta=$ $20.77,21.23,24.99,27.70,31.04$ (C-2, C-3, C-4, C-5, $\mathrm{C}-6), 28.86\left(\mathrm{CH}_{3} \mathrm{CO}\right), 86.22,86.60\left(\mathrm{C}-2^{\prime}, \mathrm{C}-3^{\prime}\right), 118.06$ $\left(\mathrm{C}-8^{\prime}\right), 119.35\left(\mathrm{C}-6^{\prime}, 122.15\left(\mathrm{C}-4 \mathrm{a}^{\prime}\right), 128.54\left(\mathrm{C}-5^{\prime}\right), 136.35\right.$

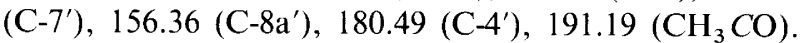
MS (EI): $m / z(\%) 292\left(M-\mathrm{S}_{2}, 70\right), 250$ (100), 207 (67), 121 (22), 43 (51). The FAB spectrum exhibits a significant $\mathrm{MH}^{+}$ion at $m / z 357$ as well as ions at $m / z 292,250$, and 207, all including chlorine as indicated by their isotopic patterns. Found: C, 53.95; H, 4.69; $\mathrm{Cl}, 10.03 ; \mathrm{S}$, 17.79; calc. for $\mathrm{C}_{16} \mathrm{H}_{17} \mathrm{ClO}_{3} \mathrm{~S}_{2}$ (356.87): C, 53.84; $\mathrm{H}$, $4.80 ; \mathrm{Cl}, 9.93 ; \mathrm{S}, 17.96 \%$.

Acetyl 3-chloro-4-oxo-2,2,6-trimethylthiochroman-3-yl disulfide 11e. M.p. $100-102{ }^{\circ} \mathrm{C}$ (from ether-petroleum ether, b.p. $40-60{ }^{\circ} \mathrm{C}$ ), yield $89 \%$. IR $(\mathrm{KBr}): v_{\mathrm{C}=\mathrm{O}}=$ $1701 \mathrm{~cm}^{-1}$. ${ }^{1} \mathrm{H}$ NMR $(250 \mathrm{MHz}): \delta=1.61 \quad(3 \mathrm{H}, \mathrm{s}$, $\left.2-\mathrm{Me}_{\mathrm{a}}\right), 1.85$ ( $\left.3 \mathrm{H}, \mathrm{s}, 2-\mathrm{Me}_{\mathrm{b}}\right), 2.38$ ( $\left.3 \mathrm{H}, \mathrm{s}, 6-\mathrm{Me}\right), 2.41$ $\left(3 \mathrm{H}, \mathrm{s}, \mathrm{CH}_{3} \mathrm{CO}\right), 7.12(1 \mathrm{H}, \mathrm{d}, \mathrm{ArH}), 7.25-7.31(1 \mathrm{H}$, $\mathrm{m}, \mathrm{ArH}), \quad{ }^{7.95-8.00}(1 \mathrm{H}, \mathrm{m}, \mathrm{ArH}) .{ }^{13} \mathrm{C}$ NMR $(125.7 \mathrm{MHz}): \delta=20.79\left(2-\mathrm{Me}_{\mathrm{a}}\right), 25.12,25.26\left(2-\mathrm{Me}_{\mathrm{b}}\right.$, 6-Me), 52.39 (C-2), 91.76 (C-3), 126.90 (C-6), 127.85 (C-8), 131.38 (C-5), 133.78 (C-8a), 134.83 (C-7), 135.70 (C-4a), $181.20(\mathrm{C}-4), 191.38\left(\mathrm{CH}_{3} \mathrm{CO}\right)$. MS: $m / z(\%) 346$ $(M, 3.4), 282\left(M-\mathrm{S}_{2}, 57\right), 267\left(m / z 282-\mathrm{CH}_{3}, 6\right), 247$ $(\mathrm{m} / z 282-\mathrm{Cl}, 7), 240(14), 239(9), 225\left(\mathrm{C}_{11} \mathrm{H}_{10} \mathrm{ClOS}\right.$, 100), 205 (65), 204 (70), 121 (29), 43 (54). Found: C, $48.81 ; \mathrm{H}, 4.35 ; \mathrm{Cl}, 10.02 ; \mathrm{S}, 27.61$; calc. for $\mathrm{C}_{14} \mathrm{H}_{15} \mathrm{ClO}_{2} \mathrm{~S}_{3}$ (346.89): C, 48.47; H, 4.35; Cl, 10.21; S, 27.72\%.

Reaction of 11 with morpholine. The following 'unzipping' reactions were carried out according to a literature procedure for the generation of $\mathbf{1} / 2 .^{3}$ Disulfide 11 ( $2.3 \mathrm{~g}$, $7 \mathrm{mmol}$ ) was dissolved in $50 \mathrm{ml}$ ether and treated, with stirring, with $6.0 \mathrm{ml}(60 \mathrm{mmol})$ morpholine, dissolved in $30 \mathrm{ml}$ ether. The rate of the addition was adjusted so as to avoid any appreciable rise in temperature. The reaction mixture was then extracted three times with water, dried over anhydrous $\mathrm{CaCl}_{2}$, and evaporated in vacuo. The oily residue was separated by column chromatography (aluminium oxide Merck 90, particle size 0.063$0.200 \mathrm{~mm}$; ether-hexane 1:5). For each reaction the products are described in the order of their elution from the chromatographic column.

3,5' - Diethyl - 3,5' - dimethyl - 3,4,5',6' - tetrahydrospiro \{naphthalene - 2,2' - naphtho[2,1-e][1,3,4] oxadithiin $\}-1$ one 14a. M.p. $113-115^{\circ} \mathrm{C}$. IR ( $\left.\mathrm{KBr}\right): v_{\mathrm{C}=\mathrm{O}}=1696 \mathrm{~cm}^{-1}$. MS: $m / z(\%) 436(M, 16), 404(M-\mathrm{S}, 4), 372\left(M-\mathrm{S}_{2}\right.$, 14), $218\left(\mathrm{C}_{13} \mathrm{H}_{14} \mathrm{OS}, 100\right), 185(80), 176$ (47), 118 (87), 115 (32), 90 (60). The sample size was insufficient for NMR spectroscopy and elemental analysis. 
trans - 3,3" - Diethyl - 1,1",2,2",3,3",4,4" - octahydro - 3,3" methyldispiro \{naphthalene - 2,3'- [1,2,4] trithiolane - $5^{\prime}, 2^{\prime \prime}$ naphthalene $\}-1,1^{\prime \prime}$-dione trans-15a. M.p. $162-165^{\circ} \mathrm{C}$. IR (KBr): $v_{\mathrm{C}=\mathrm{O}}=1687 \mathrm{~cm}^{-1} .{ }^{1} \mathrm{H}$ NMR $(250 \mathrm{MHz}): \delta=$ 0.78-0.89 (6 H, m, 3- $\mathrm{CH}_{3} \mathrm{CH}_{2}, 3$ " $\left.-\mathrm{CH}_{3} \mathrm{CH}_{2}\right), 1.02(6 \mathrm{H}$, t, 3-Me, 3"-Me), 1.21-1.41 (2 H, m, 3- $\mathrm{CH}_{3} \mathrm{CH}_{\mathrm{a}} \mathrm{H}_{\mathrm{b}}$, $\left.3^{\prime \prime}-\mathrm{CH}_{3} \mathrm{CH}_{\mathrm{a}} \mathrm{H}_{\mathrm{b}}\right), 1.51-1.65\left(2 \mathrm{H}, \mathrm{m}, 3-\mathrm{CH}_{3} \mathrm{CH}_{\mathrm{a}} H_{\mathrm{b}}\right.$, 3"- $\left.\mathrm{CH}_{3} \mathrm{CH}_{\mathrm{a}} \mathrm{H}_{\mathrm{b}}\right), 3.05\left(2 \mathrm{H}, \mathrm{d}, 4-\mathrm{H}_{\mathrm{a}}, 4^{\prime \prime}-\mathrm{H}_{\mathrm{a}}\right), 3.05(2 \mathrm{H}$, d, 4- $\left.\mathrm{H}_{\mathrm{b}}, 4^{\prime \prime}-\mathrm{H}_{\mathrm{b}}\right), 7.12-7.21(2 \mathrm{H}, \mathrm{m}, 2 \mathrm{ArH}), 7.28-7.39$ (2 H, m, 2 ArH ), 7.44-7.49 (2 H, m, 2 ArH ), 8.09-8.19 $(2 \mathrm{H}, \mathrm{m}, 2 \mathrm{ArH}) .{ }^{13} \mathrm{C} \mathrm{NMR}(50.32 \mathrm{MHz}): \delta=8.31$ $\left(\mathrm{CH}_{3} \mathrm{CH}_{2}\right), 22.55\left(3-\mathrm{CH}_{3}\right), 31.45\left(\mathrm{CH}_{3} \mathrm{CH}_{2}\right), 39.28(\mathrm{C}-4$, C-4"), 50.64 (C-3, C-3"), 95.70 (C-2, C-2"), 126.57, 126.99, 128.70, 130.54, 133.53, 138.89 (C-4a, C-4a", C-5, C-5", C-6, C-6", C-7, C-7", C-8, C-8", C-8a, C-8a"), $185.95\left(\mathrm{C}-1, \mathrm{C}-1^{\prime \prime}\right)$. MS: $m / z(\%) 468(M, 13), 436(M-\mathrm{S}$, 7), $404\left(M-\mathrm{S}_{2}, 100\right), 375\left(m / z 404-\mathrm{C}_{2} \mathrm{H}_{5}, 37\right), 347$ (4), 286 (7), 250 (9), 218 (44), $186(46), 185$ (77), 157 (41), 145 (32), 128 (31), 118 (48), 90 (46). Found: C, 66.77; H, 6.43; S, 19.76; calc. for $\mathrm{C}_{26} \mathrm{H}_{28} \mathrm{O}_{2} \mathrm{~S}_{3}$ (468.67): C, 66.62; H, 6.02, S 20.52\%.

cis - 3,3" - Diethyl - 1,1",2,2",3,3",4,4" - octahydro - 3,3" - di methyldispiro \{naphthalene -2,3' - [1,2,4] trithiolane - $5^{\prime}, 2^{\prime \prime}$ naphthalene - $1,1^{\prime \prime}$-dione cis-15a. M.p. $167-170^{\circ} \mathrm{C}$ (from ethyl acetate), yield $2 \%$. IR ( KBr): $v_{\mathrm{C}=\mathrm{O}}=1696 \mathrm{~cm}^{-1} .{ }^{1} \mathrm{H}$ NMR $(250 \mathrm{MHz}): \delta=0.77-0.99\left(6 \mathrm{H}, \mathrm{m}, 3-\mathrm{CH}_{3} \mathrm{CH}_{2}\right.$, 3"- $\left.\mathrm{CH}_{3} \mathrm{CH}_{2}\right), \quad 1.19-1.72 \quad\left(4 \mathrm{H}, \quad \mathrm{m}, \quad 3-\mathrm{CH}_{3} \mathrm{CH}_{2}\right.$, 3"- $\left.\mathrm{CH}_{3} \mathrm{CH}_{2}\right), 2.21\left(6 \mathrm{H}, \mathrm{s}, 2 \times 3-\mathrm{CH}_{3}\right), 2.92-3.29(4 \mathrm{H}$, $4-\mathrm{H}_{\mathrm{a}}, 4-\mathrm{H}_{\mathrm{b}}, 4$ " $-\mathrm{H}_{\mathrm{a}}, 4$ 4"- $\left.\mathrm{H}_{\mathrm{b}}\right), 7.13(2 \mathrm{H}, \mathrm{d}, 2 \mathrm{ArH})$, 7.23-7.39 (2 H, m, 2 ArH ), 7.45-7.52 (2 H, m, 2 ArH ), 8.11-8.22 (2 H, m, 2 ArH). ${ }^{13} \mathrm{C} \mathrm{NMR} \mathrm{(125.7} \mathrm{MHz):} \delta=$ $8.49\left(3-\mathrm{CH}_{3} \mathrm{CH}_{2}, 3^{\prime \prime}-\mathrm{CH}_{3} \mathrm{CH}_{2}\right), 21.71\left(3-\mathrm{CH}_{3}, 3^{\prime \prime}-\mathrm{CH}_{3}\right)$, $29.91\left(3-\mathrm{CH}_{3} \mathrm{CH}_{2}, 3^{\prime \prime}-\mathrm{CH}_{3} \mathrm{CH}_{2}\right), 38.89(\mathrm{C}-4, \mathrm{C}-4$ "), 45.63 (C-3, C-3"), 95.87 (C-2, C-2"), 126.83, 128.41, 128.69, $128.98,133.31,138.81\left(\mathrm{C}-4 \mathrm{a}+\mathrm{C}-4 \mathrm{a}^{\prime \prime}, \mathrm{C}-5+\mathrm{C}-5^{\prime \prime}, \mathrm{C}-6+\mathrm{C}-\right.$ 6", C-7 + C-7", C-8 + C-8", C-8a +C-8a"), 206.69 (C-1, C-1"). MS: $m / z(\%) 468(M, 8), 404\left(M-\mathrm{S}_{2}, 100\right), 375$ $\left(m / z 404-\mathrm{C}_{2} \mathrm{H}_{5}, 40\right), 347$ (3), 286 (4), 250 (3), 218 (18), 186 (39), 185 (58), 157 (32), 145 (23), 128 (23), 118 (28), 90 (25). Found: C, 67.01; H, 6.19; S, 19.86; calc. for $\mathrm{C}_{26} \mathrm{H}_{28} \mathrm{O}_{2} \mathrm{~S}_{3}$ (468.67): C, 66.62; H, 6.02, $\mathrm{S}$ $20.52 \%$.

trans - $1,1^{\prime \prime}, 2,2^{\prime \prime}, 3,3^{\prime \prime}, 4,4^{\prime \prime}$ - octahydro - 3,3,3",3",5,5",8, $8^{\prime \prime}$ octamethyldispiro \{naphthalene - 2,3' - [1,2,4] trithiolane 5',2"-naphthalene $\}-1,1^{\prime \prime}$-dione trans-15b. M.p. 218-220 ${ }^{\circ} \mathrm{C}$. IR $(\mathrm{KBr}): v_{\mathrm{C}=\mathrm{o}}=1684 \mathrm{~cm}^{-1} .{ }^{1} \mathrm{H}$ NMR $(250 \mathrm{MHz}): \delta=$ $1.35\left(12 \mathrm{H}, \mathrm{s}, 3-\mathrm{Me}_{\mathrm{a}}, 3-\mathrm{Me}_{\mathrm{b}}, 3^{\prime \prime}-\mathrm{Me}_{\mathrm{a}}, 3\right.$ 3"- $\left.\mathrm{Me}_{\mathrm{b}}\right), 2.18(6 \mathrm{H}$, s, 5- $\left.\mathrm{CH}_{3}, 5^{\prime \prime}-\mathrm{CH}_{3}\right), 2.58\left(6 \mathrm{H}, \mathrm{s}, 8-\mathrm{CH}_{3}, 8^{\prime \prime}-\mathrm{CH}_{3}\right), 2.84$ ( $\left.2 \mathrm{H}, \mathrm{d}, 4-\mathrm{H}_{\mathrm{a}}, 4^{\prime \prime}-\mathrm{H}_{\mathrm{a}}\right), 2.84\left(2 \mathrm{H}, \mathrm{d}, 4-\mathrm{H}_{\mathrm{b}}, 4^{\prime \prime}-\mathrm{H}_{\mathrm{b}}\right), 7.05$ (2 H, d, 6-H, 6"-H, ArH), 7.21 (2 H, d, 7-H, 7"-H, $\mathrm{ArH}) .{ }^{13} \mathrm{C}$ NMR $(125.7 \mathrm{MHz}): \delta=19.36\left(3-\mathrm{CH}_{3}\right.$ or $\left.3^{\prime \prime}-\mathrm{CH}_{3}\right), 22.40\left(3-\mathrm{CH}_{3}\right.$ or 3 " $\left.-\mathrm{CH}_{3}\right), 26.49\left(5-\mathrm{CH}_{3}\right.$, $\left.5^{\prime \prime}-\mathrm{CH}_{3}\right), 27.36\left(8-\mathrm{CH}_{3}, 8\right.$ " $\left.-\mathrm{CH}_{3}\right), 40.28(\mathrm{C}-4, \mathrm{C}-4$ "), 43.35 (C-3, C-3"), 95.49 (C-2, C-2"), 129.65, 130.02, 133.58, 133.74, 137.80, 139.44 (C-4a + C-4a", C-5 + C-5", C-6+C-6", C-7 + C-7", C-8 + C-8", C-8a +C-8a"), 189.52
(C-1 +C-1"). MS: $m / z(\%) 496(M, 11), 464(M-\mathrm{S}, 7)$, $432\left(M-\mathrm{S}_{2}, 100\right), 417\left(\mathrm{~m} / z 432-\mathrm{CH}_{3}, 6\right), 404(4), 389$ (3), $286(6), 232$ (23), 217 (21), 200 (29), 185 (18), 173 (20), 146 (30), 117 (25). Found: C, 67.66; H, 6.93; S, 19.27; calc. for $\mathrm{C}_{28} \mathrm{H}_{32} \mathrm{O}_{2} \mathrm{~S}_{3}$ (496.73): C, 67.69; H, 6.49; S, $19.36 \%$.

trans - 1,1", 2,2",3,3",4,4" - Octahydro-3,3,3",3" - tetraethyldispiro \{naphthalene - 2,3' - [1,2,4] trithiolane - 5',2" naphthalene $\}-1,1^{\prime \prime}$-dione trans-15c. M.p. $190-192^{\circ} \mathrm{C}$ (from petroleum ether, b.p. $40-60^{\circ} \mathrm{C}$ ). IR $(\mathrm{KBr}): v_{\mathrm{C}=\mathrm{O}}=$ $1695 \mathrm{~cm}^{-1}$. ${ }^{1} \mathrm{H}$ NMR $(250 \mathrm{MHz}): \delta=0.89\left(6 \mathrm{H}, \mathrm{t}, 3-\mathrm{Me}_{\mathrm{a}}\right.$, 3"- $\left.\mathrm{Me}_{\mathrm{a}}\right), 1.11\left(6 \mathrm{H}, \mathrm{t}, 3-\mathrm{Me}_{\mathrm{b}}, 3^{\prime \prime}-\mathrm{Me}_{\mathrm{b}}\right), 1.85-2.28(8 \mathrm{H}$, $\left.\mathrm{m}, 2 \times 3-\mathrm{CH}_{3} \mathrm{CH}_{2}, 2 \times 3^{\prime \prime}-\mathrm{CH}_{3} \mathrm{CH}_{2}\right), 3.15\left(4 \mathrm{H}, \mathrm{s}, 4-\mathrm{CH}_{2}\right.$, 4"- $\left.\mathrm{CH}_{2}\right), 7.15-7.57,8.17(8 \mathrm{H}, \mathrm{m}, 8 \mathrm{ArH}) .{ }^{13} \mathrm{C}$ NMR $(125.7 \mathrm{MHz}): \delta=9.69, \quad 9.98 \quad\left(2 \times 3-\mathrm{CH}_{3} \mathrm{CH}_{2}+2 \times 3 "-\right.$ $\left.\mathrm{CH}_{3} \mathrm{CH}_{2}\right), \quad 28.32, \quad 29.59 \quad\left(2 \times 3-\mathrm{CH}_{3} \mathrm{CH}_{2}+2 \times 3^{\prime \prime}-\right.$ $\left.\mathrm{CH}_{3} \mathrm{CH}_{2}\right), 39.51\left(\mathrm{C}-4+\mathrm{C}-4^{\prime \prime}\right), 47.48\left(\mathrm{C}-3+\mathrm{C}-3{ }^{\prime \prime}\right), 96.70$ $\left(\mathrm{C}-2+\mathrm{C}-2^{\prime \prime}\right), 126.98,128.59,128.67,130.77,133.29$, 139.02 (C-4a + C-4a", C-5 + C-5", C-6 + C-6", C-7 + C-7", $\mathrm{C}-8+\mathrm{C}-8$ ", C-8a + C-8a"), $186.73\left(\mathrm{C}-1+\mathrm{C}-1^{\prime \prime}\right)$. MS: $m / z$ (\%) $496(M, 8), 464(M-\mathrm{S}, 1), 432\left(M-\mathrm{S}_{2}, 100\right), 375$ (3), 314 (3), 285 (2), 264 (8), 232 (14), 217 (7), 200 (35), 199 (47), 171 (24), 159 (14), 118 (11), 90 (11). Found: C, 67.57, H, 6.67, S, 18.80; calc. for $\mathrm{C}_{28} \mathrm{H}_{32} \mathrm{O}_{2} \mathrm{~S}_{3}$ (496.73): C, 67.69, H, 6.49, S, 19.36\%.

cis - 1,1",2,2",3,3",4,4" - Octahydro - 3,3,3",3" - tetraethyl dispiro \{naphthalene $-2,3^{\prime}-[1,2,4]$ trithiolane $-5^{\prime}, 2^{\prime \prime}$-naphthalene - $1,1^{\prime \prime}$-dione cis-15c. M.p. $148-150^{\circ} \mathrm{C}$ (from petroleum ether, b.p. $\left.40-60^{\circ} \mathrm{C}\right)$. IR ( $\left.\mathrm{KBr}\right): v_{\mathrm{C}=\mathrm{O}}=1693 \mathrm{~cm}^{-1} \cdot{ }^{1} \mathrm{H}$ NMR (250 MHz): $\delta=0.90\left(6 \mathrm{H}, \mathrm{t}, 3-\mathrm{Me}_{\mathrm{a}}, 3 "-\mathrm{Me}_{\mathrm{a}}\right), 1.06$ $\left(6 \mathrm{H}, \mathrm{t}, 3-\mathrm{Me}_{\mathrm{b}}, 3^{\prime \prime}-\mathrm{Me}_{\mathrm{b}}\right), 1.69-2.21(8 \mathrm{H}, \mathrm{m}, 2 \times 3-$ $\left.\mathrm{CH}_{3} \mathrm{CH}_{2}, 2 \times 3^{\prime \prime}-\mathrm{CH}_{3} \mathrm{CH}_{2}\right), 3.05\left(2 \mathrm{H}, \mathrm{d}, 4-\mathrm{CH}_{\mathrm{a}} \mathrm{H}_{\mathrm{b}}, 4\right.$ "$\left.\mathrm{CH}_{\mathrm{a}} \mathrm{H}_{\mathrm{b}}\right), 3.17\left(2 \mathrm{H}, \mathrm{d}, 4-\mathrm{CH}_{\mathrm{a}} H_{\mathrm{b}}, 4^{\prime \prime}-\mathrm{CH}_{\mathrm{a}} H_{\mathrm{b}}\right), 7.15-7.20$ (2 H, dd, $2 \mathrm{ArH}), 7.22-7.38$ (2 H, m, $2 \mathrm{ArH}), 7.41-7.52$ ( 2 H, dd, $2 \mathrm{ArH}), 8.11-8.19$ ( $2 \mathrm{H}, \mathrm{dd}, 2 \mathrm{ArH}) .{ }^{13} \mathrm{C}$ NMR $(125.7 \mathrm{MHz}): \delta=9.66,9.90 \quad\left(2 \times 3-\mathrm{CH}_{3} \mathrm{CH}_{2}, \quad 2 \times 3 "-\right.$ $\left.\mathrm{CH}_{3} \mathrm{CH}_{2}\right), 27.98\left(2 \times 3-\mathrm{CH}_{3} \mathrm{CH}_{2}, \quad 2 \times 3^{\prime \prime}-\mathrm{CH}_{3} \mathrm{CH}_{2}\right)$, 38.91 (C-4, C-4"), 47.81 (C-3, C-3"), 95.66 (C-2, C-2"), $126.88,128.50,128.69,130.98,133.35,138.94$ (C-4a, C-4a", C-5, C-5", C-6, C-6", C-7, C-7", C-8, C-8", C-8a, C-8a"), 186.73 (C-1, C-1"). MS: $m / z$ (\%) identical with the above-mentioned EI spectrum of trans-15c. Found: $\mathrm{C}, 67.67, \mathrm{H}, 6.73, \mathrm{~S}, 18.84$; calc. for $\mathrm{C}_{28} \mathrm{H}_{32} \mathrm{O}_{2} \mathrm{~S}_{3}$ (496.73): C, 67.69, H, 6.49, S, $19.36 \%$.

trans-Tetraspiro \{cyclohexane-1,2'-chroman-3',3"-[1,2,4]trithiolane -5", $3^{\prime \prime \prime}$ - chroman - $2^{\prime \prime \prime}, 1^{\prime \prime \prime \prime}$ - cyclohexane $\}-4^{\prime}, 4^{\prime \prime \prime}$ dione trans-15d. M.p. $280-282^{\circ} \mathrm{C}$ (from ether). IR (KBr): $v_{\mathrm{C}=\mathrm{O}}=1697 \mathrm{~cm}^{-1}$. ${ }^{1} \mathrm{H}$ NMR $(250 \mathrm{MHz}): \delta=$ $1.18-1.59\left(8 \mathrm{H}, \mathrm{m}, 3-\mathrm{CH}_{2}, 3^{\prime \prime \prime \prime}-\mathrm{CH}_{2}, 5-\mathrm{CH}_{2}, 5^{\prime \prime \prime \prime}-\mathrm{CH}_{2}\right)$, 1.61-1.98, 2.17-2.45 (8 H, m, m, 2- $\mathrm{CH}_{2}, 2^{\prime \prime \prime \prime}-\mathrm{CH}_{2}$, 6- $\left.\mathrm{CH}_{2}, 6^{\prime \prime \prime \prime}-\mathrm{CH}_{2}\right), 2.59-2.85\left(4 \mathrm{H}, \mathrm{m}, 4-\mathrm{CH}_{2}, 4^{\prime \prime \prime \prime}-\mathrm{CH}_{2}\right)$, 6.98-7.09 (2 H, m, 2 ArH), 7.27 (2 H, s, $2 \mathrm{ArH})$, 7.49-7.59 (2 H, m, $2 \mathrm{ArH}), 7.98-8.07$ ( $2 \mathrm{H}, \mathrm{dd}, 2 \mathrm{ArH})$. ${ }^{13} \mathrm{C}$ NMR $(62.9 \mathrm{MHz}): \delta=21.00,21.83,25.16,31.64$, 32.63 (C-2 + C-2 $2^{\prime \prime \prime \prime}$, C-3 + C-3 $3^{\prime \prime \prime \prime}$, C-4 + C-4'"', C-5 + C-5 $5^{\prime \prime \prime \prime}$, 
C-6 + C-6'"') $), 85.18\left(\mathrm{C}-2^{\prime}+\mathrm{C}-2^{\prime \prime}\right), 91.71\left(\mathrm{C}-3^{\prime}+\mathrm{C}-3^{\prime \prime}\right)$, $118.01,119.53,121.94,128.15,135.85,156.55\left(\mathrm{C}-4 \mathrm{a}^{\prime}+\mathrm{C}-\right.$ $4 a^{\prime \prime \prime}$, C-5' + C-5"', C-6' + C-6"', C-7' + C-7"', C-8 + C-8"', $\left.\mathrm{C}-8 \mathrm{a}^{\prime}+\mathrm{C}-8 \mathrm{a}^{\prime \prime}\right), 182.21\left(\mathrm{C}-4^{\prime}+\mathrm{C}-4^{\prime \prime}\right)$. MS: $m / z(\%): 524$ $(M, 100), 492(M-\mathrm{S}, 2), 460\left(M-\mathrm{S}_{2}, 10\right), 404(11), 340$ (13), 247 (10), 213 (22), 201 (60), 126 (22), 121 (25). Found: C, 63.87, H, 5.49, S, 18.34; calc. for $\mathrm{C}_{28} \mathrm{H}_{28} \mathrm{O}_{4} \mathrm{~S}_{3}$ (524.68): C, 64.09, H, 5.38, S, 18.33\%.

trans - 2,2,2",2",6,6" - Hexamethyldispiro \{thiochroman $3,3^{\prime}-[1,2,4]$ trithiolane - 5',3" - thiochroman $\}-4,4^{\prime \prime}$ - dione trans-15e. M.p. $198-201{ }^{\circ} \mathrm{C}$ (from petroleum ether, b.p. $\left.40-60{ }^{\circ} \mathrm{C}\right)$. IR $(\mathrm{KBr}): v_{\mathrm{C}=\mathrm{O}}=1693 \mathrm{~cm}^{-1} .{ }^{1} \mathrm{H} \quad \mathrm{NMR}$ $(250 \mathrm{MHz}): \delta=1.59\left(6 \mathrm{H}, \mathrm{s}, 2-\mathrm{Me}_{\mathrm{a}}+2^{\prime \prime}-\mathrm{Me}_{\mathrm{a}}\right), 2.15(6 \mathrm{H}$, $\left.\mathrm{s}, \quad 2-\mathrm{Me}_{\mathrm{b}}+2^{\prime \prime}-\mathrm{Me}_{\mathrm{b}}\right), 2.35\left(6 \mathrm{H}, \quad \mathrm{s}, \quad 6-\mathrm{Me}+6^{\prime \prime}-\mathrm{Me}\right)$, 7.05-7.28 (4 H, m, $4 \mathrm{ArH}), 8.09(2 \mathrm{H}, \mathrm{d}, 2 \mathrm{ArH}) .{ }^{13} \mathrm{C}$ NMR $(50.32 \mathrm{MHz}): \delta=20.87\left[\left(2-\mathrm{CH}_{3}\right)_{\mathrm{a}}+\left(2{ }^{\prime \prime}-\mathrm{CH}_{3}\right)_{\mathrm{a}}\right]$, $26.53\left[\left(2-\mathrm{CH}_{3}\right)_{\mathrm{b}}+\left(2^{\prime \prime}-\mathrm{CH}_{3}\right)_{\mathrm{b}}\right], 28.19\left(6-\mathrm{CH}_{3}+6^{\prime \prime}-\mathrm{CH}_{3}\right)$, $51.55\left(\mathrm{C}-2+\mathrm{C}-2^{\prime \prime}\right), 98.57\left(\mathrm{C}-3+\mathrm{C}-3^{\prime \prime}\right), 126.86,128.16$, 131.17, 134.32, 134.45, 135.65 (C-4a + C-4a", C-5 + C-5", C-6 + C-6", C-7+C-7", C-8 +C-8", C $\left.8 \mathrm{a}+\mathrm{C}-8 \mathrm{a}^{\prime \prime}\right), 183.40$ $\left(\mathrm{C}-4+\mathrm{C}-4^{\prime \prime}\right)$. MS: $m / z(\%) 504(M, 16), 472(M-\mathrm{S}, 3)$, $440\left(M-\mathrm{S}_{2}, 2\right), 425(3), 334$ (22), 290 (8), $236(22)$, 204 (100), 150 (52), 121 (13). Found: C, 57.21, H, 4.64, S, 31.77; calc. for $\mathrm{C}_{24} \mathrm{H}_{24} \mathrm{O}_{2} \mathrm{~S}_{5}$ (504.73): C, 57.10, H, 4.79, S, 31.76\%.

Acknowledgements. A grant from the Egyptian Government under the Channel System for M. I. Hegab is gratefully acknowledged. This work was also supported by the Danish Research Council (SNF). The technical support of Mrs. Jytte Grove-Rasmussen, Department of Organic Chemistry, Technical University of Denmark is gratefully acknowledged.

\section{References}

1. Huisgen, R. and Rapp, J. Tetrahedron 53 (1997) 939, and references cited therein.

2. Ishii, A., Akazawa, T., Ding, M.-X., Honjo, T., Maruta, T., Nakamura, S.-Y., Nagaya, H., Ogura, M., Teramoto, K., Shiro, M., Hoshino, M. and Nakayama, J. Bull. Chem. Soc. Jpn. 70 (1997) 509, and literature cited therein.

3. Fabian, J. and Senning, A. Sulfur Rep. 21 (1998) 1, and literature cited therein.

4. Senning, A., Hansen, H. C., Abdel-Megeed, M. F., Mazurkiewicz, W. and Senning, A. Tetrahedron 42 (1986) 739.

5. Franek, W. Monatsh. Chem. 127 (1996) 895, 909.

6. Bell, V. L. and Cromwell, N. H. J. Org. Chem. 23 (1958) 789.

7. Prout, F. S., Hartman, R. J., Huang, E. P. Y., Korpics, C. J. and Tichelaar, G. R. Org. Synth., Coll. Vol. IV (1963) 93

8. Crossland, I. Acta Chem. Scand., Ser. B 31 (1977) 890.

9. Kabbe, H. J. Synthesis (1978) 886.

10. Clayton. S. E., Gabbutt, C. D., Hepworth. J. D. and Heron. B. M. Tetrahedron 49 (1993) 939.

11. Crossland, I. Acta Chem. Scand., Ser. B 30 (1976) 787.

12. Gabbutt, C. D., Hepworth, J. D. and Heron, B. M. Tetrahedron 50 (1994) 5245.

13. Kelly, S. E. and Vanderplas, B. C. J. Org. Chem. 56 (1991) 1325.

14. Cowan, D. M. and Vogel, A. I. J. Chem. Soc. (1940) 1528.

Received July 27, 1998. 